\title{
ПРИМЕНЕНИЕ ГАРМОНИЧЕСКОГО АНАЛИЗА ДЛЯ ИЗУЧЕНИЯ НЕСТАЦИОНАРНОГО РЕЖИМА ОДНОМЕРНОЙ ФИЛЬТРАЦИИ ПОТОКА ПОДЗЕМНЫХ ВОД СО СВОБОДНОЙ ПОВЕРХНОСТЬЮ
}

В связи с широким внедрением быстродействующей вычислительной техники наступила возможность использовать такие методы исследования природных процессов, практическое прилсжение которых раньше считалось слишком громоздким или даже неосуществимым.

В статье предлагается применить гармонический анализ для нзучения нестационарного режима одномерной фильтрации потока подземных вод со свободной поверхностью.

\section{Постановка проблемы}

Нестационарное распределение гидравлического напора в горизонте подземных вод со свободной поверхностью описывается, согласно теории $\left[{ }^{1-3}\right]$, дифференциальным уравнением

$$
\partial(h k \partial H / \partial x) / \partial x+\partial(h k \partial H / \partial y) / \partial y+w=\mu \partial H / \partial t,
$$

где $H(x, y, t)$ - гидравлический напор; $h(x, y, t)-$ мощность водоносного горизонта; $k(x, y)$ - коэффициент фильтрации; $w(x, y, t, H)$ функция внутреннего источника (такими могут являться инфильтрация, испарение, подпитание из нижележащих водоносных горизонтов и т. п.); $\mu$ - при подпоре $(\partial H / \partial t>0)$ соответствует коэффициенту недостатка. насыщения $\mu_{\mathrm{H}}$, при спаде $(\partial H / \partial t<0)$ - коэффициенту водоотдачи $\mu_{\mathrm{B}}$.

Уравнение (1) нелинейное, так как величины $h$, напора $H$.

Если рассматривать однородный водовмещающий пласт, внутренний источник в котором отсутствует $(w=0)$, залегающий на горизонтальном водоупорном основании, причем распределение напора зависит только от одной пространственной координаты, то $H=h ; k(x, y)=\bar{k}$. и уравнение (1) представляется в упрощенном виде:

$$
\bar{k} \partial(h \partial h / \partial x) / \partial x=\mu \partial h / \partial t .
$$

Требуется аналитически найти функцию распределения мощности водоносного горизонта $h(x, t)$ в конечном интервале изменений

$$
0 \leqslant x \leqslant l
$$

при граничных условиях

$$
h(0, t)=h_{0}(t), \quad h(l, t)=h_{l}(t)
$$


и начальном условии

$$
h(x, 0)=\left\{h_{0}^{2}(0)+\left[h_{l}^{2}(0)-h_{0}^{2}(0)\right] x / l\right\}^{1 / 2},
$$

где $l$ - длина изучаемого потока *.

Здесь принято, что поток грунтовых вод формируется за счет разности уровней смежных поверхностных водоемов (реки, каналы, водохранилища и т. п.) и движется преимущественно по направлению увеличения значений $x$ (см. рисунок). Уровни поверхностных водоемов в сечениях потока $x=0$ и $x=l$ подвергаются колебаниям, вызванным природными или техническими факторами. Колебания уровней являются некоторыми функциями от времени, соответственно $h_{0}(t)$ и $h_{l}(t)$.

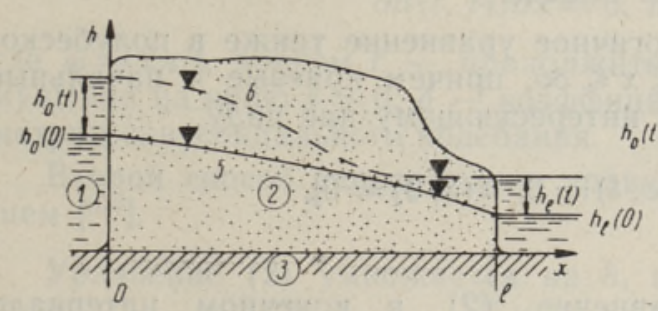

б

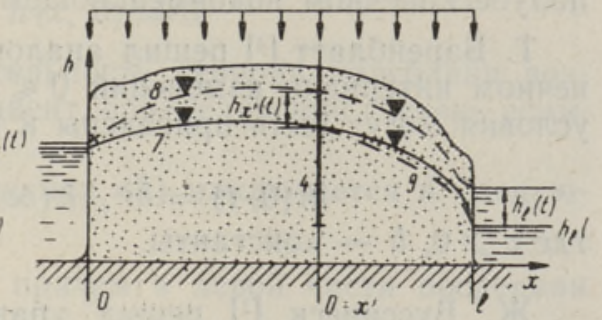

Схемы формирования потока подземных вод со свободной поверхностью:

$a-$ при $w=0$. б - при $w>0 ; t$ - поверхностный водоем, 2 - водовмещающиі пласт, 3 - водоупорное основание, 4 - наблюдательный колодец, 5 - поверхность подземных вод при $t=0 ; 6-$ то же при $t>0 ; 7-$ то же при $t=0 ; w=w_{1}>0 ; 8-$ то же при $t>0 ; w \approx 2 w_{1} ; 9-$ аппроксимирующая поверхность при $t=0$.

Начальное условие (5) принято на основе предположения, что при $t \leqslant 0$ подземный поток подчиняется стационарному режиму фильтрации $\left[h_{0}(t), h_{l}(t)=\right.$ const $]$ и распределение его мощности при этом можно описать известной в гидрогеологии параболической кривой депрессии Дюпюи $\left[{ }^{2}, 3\right]$.

Для изучения динамики стока грунтовых вод в любом сечении горизонта следует найти мәновенный расход потока**

$$
q(x, t)=\bar{k} h(x, t) \partial h(x, t) / \partial x .
$$

Суммарный расход потока за промежуток времени от 0 до $t$ представляется в таком случае интегралом

$$
Q(x, t)=\bar{k} \int_{0}^{t}[h(x, \Theta) \partial h(x, \Theta) / \partial x] d \Theta .
$$

Необходимость определения значений $h(x, t), q(x, t)$ и $Q(x, t)$ может возникать при решении различных проблем гидротехнического строительства и мелиорации. Выяснение закономерностей изменения $q(x, t)$ и $Q(x, t)$ важно при изучении вопросов формирования естественных ресурсов подземных вод.

* Приближенное решение уравнения (2) при заданных условиях (3) - (5) можно найти при помощи известных численных методов (метод сеток, метод прямых и др.). Мы предпочитаем пользоваться аналитическим методом, поскольку полученное таким образом решение более наглядно и имеет преимущество с точки зрения последующих теоретических исследований проблем режима подземных вод.

** Следует иметь в виду, что реальное содержание имеют только абсолютные зна.ения $q$, т. е. $|q|$. Однако знак перед правой стороной формулы (6) показывает направление подземного стока. При отрицательных значениях $\partial h(x, t) / \partial x$ подземные воды перемещаются в направлении возрастающих значений $x$, при положительных значениях сток происходит в направлении убывающих значений $x$. 


\section{Предыдущие исследования}

Целесообразно рассмотреть существующие характерные методы аналитического решения уравнения (2) применительно к гидрогеологическим задачам.

Т очны е решен и я. П. Полубариновой-Кочиной [3] даны точные решения уравнения (2) в полубесконечном интервале изменений $0 \leqslant x \leqslant \infty$ при условиях $\left[h(0, t)=h_{1}=\right.$ const, $\left.h(\infty, t)=0, h(x, 0)=0\right]$ и $\left[h(0, t)=0, h(\infty, t)=h_{1}, h(x, 0)=h_{1}\right]$. Эти решения соответствуют случаю мгновенного подъема или спада уровня воды в водоеме при горизонтальном начальном залегании поверхности подземных вод в полубесконечном водовмещающем пласте.

T. Баренблатт $\left.{ }^{4}\right]$ решил аналогичное уравнение также в полубесконечном интервале изменений $0 \leqslant x \leqslant \infty$, причем краевые и начальные условия могут быть приведены к интересующему нас виду

$$
\left[h(0, t)=b t^{c}, h(\infty, t)=0, h(x, 0)=0\right],
$$

где $c \geqslant 0, b-$ константы.

Ж. Буссинеск [1] решил уравнение (2) в конечном интервале $0 \leqslant x \leqslant l$ при условиях $[h(0, t)=0, \partial h(l, t) / \partial x=0, h(x, 0)=g F(x / l)]$, где $F(x / l)$ - некоторая нелинейная функция от $x$, не совпадающая с параболической зависимостью Дюпюи (5); $g$ - константа.

$\mathrm{P}$ ешения, полученные при помощи лине аризации и сходного уравнения. Один из способов линеаризации уравнения (2), называемый здесь первым, сводится к осреднению проводимости пласта $\bar{h} \bar{k}$ в пространстве и во времени [1,5]. Тогда уравнение (2) представляется в виде

$$
a \partial^{2} h / \partial x^{2}=\partial h / \partial t
$$

где $a=\bar{h} \bar{k} / \mu>0-$ коэффициент уровнепроводности; $\bar{h}-$ некоторая осредненная глубина потока подземных вод.

По мнению Н. Веригина $\left[{ }^{6,7}\right]$, описанный способ линеаризации уравнения (2) должен привести при расчетах к значительным погрешностям. По физической сущности уравнения (8) уровень грунтовых вод при приближении режима фильтрации к стационарному стремится к прямой линии, хотя на самом деле он должен представлять собой большей частью параболическую кривую.

Тем не менее в гидрогеологии известен ряд частных решений уравнения (2), линеаризованного по первому способу.

С. Аверьяновым $\left[{ }^{8}\right]$ приводятся решения применительно к следующим условиям:

$$
\begin{gathered}
{[h(0, t)=1, \partial h(l, t) / \partial t=0, h(x, 0)=0]} \\
{[h(0, t)=1, h(l, t)=0, h(x, 0)=0]} \\
{[h(0, t)=1, \partial h(l, t) / \partial x=(m / l) \partial h / \partial t, h(0, t)=1],}
\end{gathered}
$$

причем $m-$ константа. 
В. Шестаков [5] дает решения для условий

$$
\left[h(0, t)=h_{1}=\text { const, } h(l, 0)=h_{2}=\right.\text { const }
$$

$$
\left.h(l, t)=h_{3}=\text { const, } h(x, 0)=h_{1}+\left(h_{2}-h_{1}\right) x / l\right]
$$

$\left[\partial h(0, t) / \partial h=0, h(l, t)=h_{2}=\right.$ const, $\left.h(x, 0)=f(x)\right]$,

где $f(x)$ - линейная функция от $x$.

Решение X. Купера и М. Рорабога (Cooper, Rorabaugh) [ํ] выведено при краевых и начальных условиях

$$
h(0, t)=\left(\delta^{2}+\omega^{2}\right)(1-\cos \omega t) \exp [(2 \delta \arctan \omega / \delta) / \omega] / 2 \omega^{2}
$$

и

$$
\partial h(l, t) / \partial x=0, h(x, 0)=0,
$$

где $\omega=2 \pi / t$, причем $t-$ продолжительность периода колебания возмущения на краю $x=0 ; \delta-$ коэффициент, определяющий степень асимметрии синусоидального колебания.

Второй способ линеаризации уравнения (2) заключается в следующем $\left[{ }^{5-7}\right]$.

Уравнение (2) умножается на $h$, причем в левой части последняя заменяется на осредненную $\bar{h}$. Вводится новая переменная

$$
u=h^{2} / 2 \text {, }
$$

вследствие чего получается

$$
a \partial^{2} u / \partial x^{2}=\partial u / \partial t
$$

где $a=\bar{k} h / \mu \approx \bar{k} \bar{h} / \mu>0$.

Уравнение (11) является линейным в отношении функции $u$. Оно может быть решено при начальном условии

$$
u(x, 0)=A x+B,
$$

где $A$ и $B-$ постоянные, определяемые интегрированием уравнения

$$
a \partial^{2} u / \partial x=0
$$

которое описывает стационарный режим фильтрации. Можно принять, что $A=\left(h_{l}^{2}-h_{0}^{2}\right) / 2 l$ и $B=h_{0}^{2} / 2$, тогда

$$
h=\left[h_{0}^{2}+\left(h_{l}^{2}-h_{0}^{2}\right) x / l\right]^{1 / 2}
$$

(имеется в виду, что реальны только значения $h>0$ ).

Выражение (14) описывает свободную поверхность плоского в разрезе потока грунтовых вод при стационарном режиме фильтрации и совпадает с условием (5).

Н. Веригиным [7] дано решение уравнения (2) с применением второго описанного способа его линеаризации для условий

$$
\begin{gathered}
{\left[h(0, t)=h_{1}=\text { const, } h(l, 0)=h_{2}=\text { const, } h(l, t)=h_{3}=\right.\text { const }} \\
\left.h(x, 0)=\left[h_{1}^{2}+\left(h_{2}^{2}-h_{1}^{2}\right) x / l\right]^{1 / 2}\right] \text { и } \\
{\left[k h(0, t) \partial h(0, t) / \partial x=r=\text { const, } h(l, 0)=h_{1}=\right.\text { const }} \\
\left.h(l, t)=h_{2}=\text { const, } \quad h(x, 0)=\left(h_{1}^{2}-2 r x / k\right)^{1 / 2}\right] .
\end{gathered}
$$

Разработаны также способы изучения функции $h(x, t)$ при изменении граничного условия $h(0, t)$ в виде ступенчатого и ломаного графиков $[5,7,10]$. 


\section{Способы решения}

Для решения поставленной проблемы воспользуемся описанным выше вторым способом линеаризации уравнения (2). В соответствии с этим требуется решить уравнение (11) при граничных условиях

$$
u(0, t)=h^{2}(0, t) / 2=u_{0}(t) ; \quad u(l, t)=h^{2}(l, t) / 2=u_{l}(t)
$$

начальном условии

$$
\begin{aligned}
u(x, 0) & =\left\{h_{0}^{2}(0)+\left[h_{l}^{2}(0)-h_{0}^{2}(0)\right] x / l\right\} / 2= \\
& =u_{0}(0)+\left[u_{l}(0)-u_{0}(0)\right] x / l
\end{aligned}
$$

в конечном интервале изменений $x$ (3).

Поскольку имеется решение аналогичного уравнения при таких же краевых условиях [11], воспользуемся им и здесь. Тогда после замены обозначений и перехода к размерным величинам (исходное решение выведено в безразмерной форме) получим:

$u(x, t)=u_{0}(t)+\left[u_{l}(t)-u_{0}(t)\right] x / l+\sum_{i=1}^{\infty}\left[\left(2 l^{2} \sin i \pi x / l\right) / a i^{3} \pi^{3}\right] v_{i}(t)$, где

$$
\begin{gathered}
v_{i}(t)=\partial\left[(-1)^{i} u_{l}(t)-u_{0}(t)\right] / \partial t- \\
-\left\{\partial\left[(-1)^{i} u_{l}(0)-u_{0}(0)\right] / \partial t\right\} \exp \left[-(i \pi / l)^{2} a t\right]- \\
-\int_{0}^{t}\left\{\partial^{2}\left[(-1)^{i} u_{l}(\Theta)-u_{0}(\Theta)\right] / \partial \Theta^{2}\right\} \exp \left[a(i \pi / l)^{2}(\theta-t)\right] d \theta .
\end{gathered}
$$

Заменяя на основе соотношения (10) переменные, получим выражение, описывающее распределение мощности потока грунтовых вод *:

$$
\begin{gathered}
h(x, t)=\left\{\left[h_{0}^{2}(0)+\left(h_{l}{ }^{2}(0)-h_{0}{ }^{2}(0)\right) x / l\right]+\right. \\
\left.+\sum_{i=1}^{\infty}\left[\left(l^{2} \sin i \pi x / l\right) / 2 a i^{3} \pi^{3}\right] \chi_{i}(t)\right\}^{1 / 2},
\end{gathered}
$$

где

$$
\begin{gathered}
\chi_{i}(t)=\partial\left[(-1)^{i} h_{l}^{2}(t)-h_{0}^{2}(t)\right] / \partial t- \\
-\left\{\partial\left[(-1)^{i} h_{l}^{2}(0)-h_{0}^{2}(0)\right] / \partial t\right\} \exp \left[-(i \pi / l)^{2} a t\right]- \\
-\int_{0}^{t}\left\{\partial^{2}\left[(-1)^{i} h_{l}^{2}(\Theta)-h_{0}^{2}(\Theta)\right] / \partial \Theta^{2}\right\} \exp \left[a(i \pi / l)^{2}(\Theta-t)\right] d \theta .
\end{gathered}
$$

Имея в виду, что по формуле (6) и соотношению (10)

$$
q(x, t)=\bar{k} h(x, t) \partial h(x, t) / \partial x=\bar{k} \partial u(x, t) / \partial x,
$$

получим на основе выражений (17) - (21) формулу для мгновенного расхода потока грунтовых вод:

$$
q(x, t)=\bar{k}\left\{\left[h_{l}^{2}(t)-h_{0}^{2}(t)\right] / 2 l+\sum_{i=1}^{\infty}\left[(l \cos i \pi x / l) / a i^{2} \pi^{2}\right] \chi_{i}(t)\right\}
$$

* Здесь не учитывается наличие промежутка высачивания, что обусловлено несовершенством гидравлической теории движения грунтовых вод Дюпюи-Форхгеймера $[3.12]$. Вследствие этого действительная ордината кривой депрессии $h(x) \lim h_{l}+\varepsilon$ при $x \rightarrow l$ отличается от вычисленной по формуле (5), поскольку $\varepsilon>0$.

Формула (19) не учитывает также поднятие поверхности грунтовых вод под влиянием капиллярных сил. 
Суммарный расход потока грунтовых вод за промежуток времени от 0 до $t$ согласно формулам (7) и (22) выражается в виде

$$
\begin{gathered}
Q(x, t)=\bar{k} \int_{0}^{t}\left[h_{l}^{2}(\Theta)-h_{0}^{2}(\Theta)\right] / 2 l+ \\
\left.+\sum_{i=1}^{\infty}\left[\left(l^{2} \cos i \pi x / l\right) / a i^{2} \pi^{2}\right] \chi_{i}(\Theta)\right\} d \Theta .
\end{gathered}
$$

\section{Дополнительные соображения}

Водовмещ ающи й, пласт горизонтально-слоистого с троения. При горизонтально-слоистом строении водовмещающего пласта проницаемость является некоторой заданной функцией $k(z)$ от вертикальной координаты $z$. Доказывается $\left[{ }^{12}\right]$, что в таком случае распределение мощности водоносного горизонта описывается уравнением

Здесь

$$
\bar{a} \partial^{2} G / \partial x^{2}=\partial G / \partial t
$$

$$
G=\int_{0}^{h}(z-h) k(z) d z
$$

- вспомогательная функция, называемая потенциалом Гиринского $[12,13]$;

$$
\bar{a}=(1 / \mu h) \int_{0}^{h} k(z) d z>0 .
$$

Уравнение (24) является обобщением уравнения (2) - нетрудно убедиться, что при постоянной проницаемости пласта в вертикальном направлении $G=-k h^{2} / 2$ и $\bar{a}=\overline{k h} / \mu$, что соответствует второму описанному выше способу линеаризации уравнения (2).

Уравнение (23) линейно относительно функции $G$.

Таким образом, для определения функции распределения мощности $h(x, t)$ при горизонтально-слоистом строении водовмещающего пласта необходимо решить уравнение (23) при граничных условнях

$$
G(0, t)=G_{0}(t), \quad G(l, t)=G_{l}(t)
$$

и начальном условии

$$
G(x, 0)==G_{0}(0)+\left[G_{l}(0)-G_{0}(0)\right] x / l .
$$

Учитывая, что уравнения (11) и (24), а также краевые условия (15), (16) и (26), (27) аналогичны, можно на основе выражения (17) написать

$G(x, t)=G_{0}(t)+\left[G_{l}(t)-G_{0}(t)\right] x / l+\sum_{i=1}^{\infty}\left[\left(2 l^{2} \sin i \pi x / l\right) / a i^{3} \pi^{3}\right] \gamma_{i}(t)$, где

$$
\begin{gathered}
\gamma_{i}(t)=\partial\left[(-1)^{i} G_{l}(t)-G_{0}(t)\right] / \partial t- \\
-\left\{\partial\left[(-1)^{i} G_{l}(0)-G_{0}(0)\right] / \partial G\right\} \exp \left[-(i \pi / l)^{2} a t\right]- \\
-\int_{0}^{t}\left\{\partial^{2}\left[(-1)^{i} G_{l}(\Theta)-G_{0}(\theta)\right] / \partial \Theta^{2}\right\} \exp \left[a(i \pi / l)^{2}(\theta-t)\right] d \theta .
\end{gathered}
$$

Мощность потока подземных вод в любом сечении легко определяется при помощи графика связи $G(h)$, построенного по соотношению (25) [ $\left.{ }^{5}\right]$ - 
П̈оскольку доказано $\left[{ }^{13,14}\right]$, что

$$
q=\partial G / \partial x
$$

то на основе выражений $(6),(7),(28),(29)$ и (30) получим формулу для мгновенного расхода:

$$
q(x, t)=\left[G_{l}(t)-G_{0}(t)\right] / l+\sum_{i=1}^{\infty}\left[(2 l \cos i \pi x / l) / a i^{2} \pi^{2}\right] \gamma_{i}(t)
$$

и для суммарного расхода:

$$
Q(x, t)=\int_{0}^{t}\left\{\left[G_{l}(\Theta)-G_{0}(\Theta)\right] / l+\sum_{i=1}^{\infty}\left[(2 l \cos i \pi x / l) / a i^{2} \pi^{2}\right] \gamma_{i}(\Theta)\right\} d \Theta,
$$

применительно к условиям водовмещаюшего пласта горизонтальнослоистого строения.

Водовмещающий пласт неоднородного строения. Строго однородные или горизонтально-слоистые водовмещающие пласты встречаются в природе довольно редко. Реальные водовмещающие пласты часто являются неоднородными за счет литологической изменчивости слоев.

В таких условиях, по В. Шестакову [5], влияние нелинейности исходного дифференциального уравнения (2) не имеет серьезного значения, так как влияние колебаний уровней грунтовых вод на проводимость $h k$ оказывается меньшим, чем влияние неоднородности пласта и на проводимость, и на коэффициент уровнепроводности. Поэтому можно проводимость пласта $h k$ считать постоянной. В частности, такая предпосылка хорошо выполняется в случае пластов, сложенных аллювиальными отложениями, проницаемость которых с глубиной обычно увеличивается.

Следует также отметить, что в пластах неоднородного строения депрессионная поверхность потока подземных вод уже не соблюдает зависимости Дюпюи (5). По В. Шестакову [5], она может быть аппроксимирована без существенной погрешности прямой линией.

Следовательно, в сложных геологических условиях оправдывается применение уравнения (8) для решения фильтрационных задач [5].

Согласно вышеприведенному следует решить уравнение (8) при граничных условиях

$$
h(0, t)=h_{0}(t), \quad h(l, t)=h_{l}(t)
$$

и начальном условии

$$
h(x, 0)=h_{0}(0)+\left[h_{l}(0)-h_{0}(0)\right] x / l .
$$

В силу аналогии между уравнениями (8) и (11), а также условиями $(15),(16)$ и (33), (34) можно написать функцию распределения мощности для водовмещающего пласта неоднородного строения:

$h(x, t)=h_{0}(t)+\left[h_{l}(t)-h_{0}(t)\right] x / l+\sum_{i=1}^{\infty}\left[(2 l \sin i \pi x / l) / a i^{2} \pi^{2}\right] \varphi_{i}(t)$,

где

$$
\begin{gathered}
\varphi_{i}(t)=\partial\left[(-1)^{i} h_{l}(t)-h_{0}(t)\right] / \partial t- \\
-\left\{\partial\left[(-1)^{i} h_{l}(0)-h_{0}(0)\right] / \partial t \exp \left[-(i \pi / l)^{2} a t\right]\right\}- \\
-\int_{0}^{t}\left\{\partial^{2}\left[(-1)^{i} h_{l}(\theta)-h_{0}(\theta)\right] / \partial \Theta^{2}\right\} \exp \left[a(i \pi / l)^{2}(\theta-t)\right] d \theta
\end{gathered}
$$


Соответственно на основе выражений (6), (7) и (35) получим формулы для мгновенного и суммарного расходов потока подземных вод в неоднородном пласте:

$$
\begin{gathered}
q(x, t)=\bar{k} h(x, t)\left\{\left[h_{l}(t)-h_{0}(t)\right] / l+\sum_{i=1}^{\infty}\left[(2 l \cos i \pi x / l) / a i^{2} \pi^{2}\right] \varphi_{i}(t)\right\} \\
\text { и } Q(x, t)=\int_{0}^{t} q(x, \Theta) d \Theta .
\end{gathered}
$$

Оценка естественных ресурсов подземных вод. Естественные ресурсы представляют собой обеспеченный питанием приток или отток подземных вод; количественным их выражением является расход рассматриваемого водоносного горизонта на всей площади его распространения [14].

Для определения естественных ресурсов плоского в разрезе водоносного горизонта следовало бы решить уравнение

$$
\partial(h k \partial h / \partial x) / \partial x+w=\mu \partial h / \partial t .
$$

Здесь $w$ в сущности неизвестная функция, поскольку в настоящее время не имеется достоверных методов ее прямого определения.

Но, с другой стороны, уровень подземных вод в междуречном массиве подвергается сезонным колебаниям в соответствии с изменением инфильтрации или испарения, так как $h(x / l)$ является некоторой функцией и от $w$. Следует отметить, что при $w>0 h(x / l, w)>h(x / l)$, при $w=0 \quad h(x / l, w)=h(x / l)$ и при $\quad w<0 \quad h(x / l, w)<h(x / l)$.

Поэтому, если за начало изучаемого потока принимать некоторое новое сечение $\left(0=x^{\prime}\right)$ в области его питания, где условия сезонного изменения напора выяснены (напр., при помощи наб̆людательного колодца, см. рис. б), то, по-видимому, можно аппроксимировать уравнение (39) уравнением (2) или (24). При этом необходимо соблюдать условие, чтобы водораздел подземных вод не оказался между принятым начальным сечением потока и поверхностным водоемом.

Вычисление естественных ресурсов подземных вод производится для расчетного сечения потока $x=0$ и $x=l$ по формулам (23), (32) или (38) в зависимости от конкретных геолого-гидрогеологических условий. Последние определяют и погрешность, обусловленную аппроксимацией уравнения (39).

\section{Методы вычислений}

Хотя достоинством полученных формул (19), (22), (23), (28), (31), $(32),(35),(37)$ и (38) является возможность учитывать произвольные граничные условия, это обстоятельство вызывает и специфические осложнения при расчетах.

Природные граничные условия представляют собой в сущности непрерывные и достаточно гладкие функции времени и имеют в каждой точке производную. Но в нашем распоряжении они заданы некоторой совокупностью дискретных значений наблюдений водомерных постов, i. е. таблично. Это служит затрудняющим обстоятельством при решении с моделированием конкретных природных граничных условий, так как использование полученных формул требует вычисления производных $\partial / \partial t$ и $\partial^{2} / \partial t^{2}$ от функций $h_{0}(t), h_{l}(t), h_{0}^{2}(t), h_{l}^{2}(t), G_{0}(t)$ и $G_{l}(t)$.

Для преодоления трудностей, связанных с дифференцированием и последующим интегрированием эмпирических функций граничных усло- 
вий, рекомендуем их предварительно аппроксимировать полиномами Чебышева.

При изучении общетеоретических вопросов динамики режима фильтрационного потока желательно природные граничные условия выразить через подходящие аналитические функции. Так, например, для выяснения влияния паводков можно последние аппроксимировать периодической функцией (9).

Полученные формулы содержат функциональные ряды частично под знаком интеграла. Их вычисление вручную с необходимой точностью практически невозможно. Для выполнения этой работы целесообразно пользоваться быстродействующей вычислительной машиной.

Машинное вычисление сопровождается еще одним дополнительным преимуществом - оно позволяет включить в основную программу вычислений подпрограммы, которые надлежащим образом обрабатывают исходные данные, сглаживают в них влияние случайностей и неточностей наблюдений. Например, можно пользоваться программой для приближения эмпирической функции полиномами Чебышева $\left[{ }^{15}\right]$.

\section{ЛИТ Е РАТ У РА}

1. Boussinesq J., Recherches théoriques sur l'écoulement des nappes d'eau infiltrées dans le soil, J. math. pures et appl., Sér. 5, 10, fasc. 1 (1904).

2. Forchheimer P., Hydraulik, Leipzig u. Berlin, 1930.

3. Полубаринов а-Кочнна П. Я., Теория движения грунтовых вод, М., 1952.

4. Баренблатт Г. И., О некоторых неустановившихся движениях жидкости и газа в пористой среде, Прикл. матем. и механика, 16, вып. 1 (1952).

5. Шестаков В. М., Теоретические основы оценки подпора, водопонижения и дренажа, M., 1965.

6. Вер игин Н. Н., О неустановившемся движении грунтовых вод вблизи водохранилищ. Докл. АН СССР, нов. сер., 66, N2 6 (1949).

7. Абрамов С. К., Биндеман Н. Н., Бочевер Ф. М., Веригин Н. Н., Влияние водохранилищ на гидрогеологические условия прилегающих территорий, М., 1960.

8. Костяков А. Н., Фаворин Н. Н., Аверьянов С. Ф., Влияние оросительных систем на режим грунтовых вод, Сб. 1, М., 1956.

9. Cooper H. H., Rorabaugh M. J., Changes in ground-water movement and bank storage caused by flood waves in surface streams, Geological Survey Research, Geological Survey Professional Paper 475-B, Washington, 1963.

10. Чжан В й - и н $\mathbf{b}$, Неустановившийся режим грунтовых вод под влиянием орошения, Влияние орошения на режим грунтовых вод, Сб. ІІ, М., 1959.

11. В аллне р Л. К., Применение гармонического анализа для изучения динамики напора и стока одномерного потока подземных вод с напорной поверхностью, Изв. АН ЭССР. Химня. Геология, 16, № 2 (1967).

12. Аравин В. И., Нумеров С. Н., Теория движения жидкостей и газов в недеформируемой порнстой среде, М., 1953.

13. Ги р и нский Н. К., Комплексный потенциал потока со свободной поверхностью в пласте относительно малой мощности при $k=f(z)$, Докл. АН СССР, 51, № 5 (1946).

14. Куделин Б. И., Прннципы региональной оценки естественных ресурсов подземных вод, М., 1960.

15. Пу к К К., Приближение функции, заданной таблицей с постоянным шагом по методу наименьших квацратов при помощи ортогональных полиномов Чебышева, Программы для ЭЦВМ «Минск-2», вып. 1, Таллин, 1965.

Институт геологии

Академии наук Эстонской ССР
Поступнла в редакцию 1/IV 1967 


\section{VALLNER}

\section{HARMOONILISE ANALUUSi KASUTAMINE UHEMÕOTMELISE VABAPINNALISE MITTESTATSIONAARSE POHJAVEEVOOLU UURIMISEKS}

Vabapinnalist ühemõōtmelist mittestatsionaarset põhjaveevoolu kirjeldav mittelineaarne diferentsiaalvõrrand (2) on muutujate vahetust kasutades lähendatav lineaarsete võrranditega (8) või (11). Võrrandi (11) lahendamine raja- ning algtingimustel (15) - (16) annab avaldised pōhjaveevoolu hüdraulilise rōhu jaotuse (19), hetkelise vooluhulga (22) ning summaarse vooluhulga (23) jaoks homogeenses ja isotroopses kihis.

Girinski funktsiooni (25) abil on võimalik leida analoogilised valemid (28), (31) ja (32) horisontaalkihilise veehorisondi jaoks; korrapäratult kihilise veehorisondi puhul on vastavateks lähenditeks avaldised (35), (37) ning (38), mis saadakse vōrrandi (8) lahendamisel rajatingimustel (33)-(34).

Vabapinnalise pōhjaveevoolu looduslike ressursside arvutamiseks võib kasutada valemeid (23), (33) ja (38); selleks peab tundma põhjaveetaseme kõikumise iseloomu ühes punktis veehorisondi toitumisalal.

Empiiriliste funktsioonidena antud looduslikke rajatingimusi on soovitatav lähendada Tšebõševi polünoomidega.

\section{VALLNER}

\section{APPLICATION OF HARMONIC ANALYSIS IN THE STUDY OF THE NONSTEADY ONE-DIMENSIONAL FLOW OF UNCONFINED GROUND WATER}

The nonlinear differential equation (2) defining the nonsteady one-dimensional flow of unconfined ground water may be approximated, by changing of variables to linear equations (8) or (11). The solution of equation with boundary and initial conditions (15) and (16) yields expressions for the distribution of the hydraulic head of the ground water (19), for the flow at the given moment (22) and the summary flow (23) in a homogeneous and isotropic stratum.

With the help of Girinski's function (25) it is possible to find analogous formulas (28), (31) and (32) for a horizontally strated water horizon; in the case of an irregularly strated water horizon, the corresponding approximations are the expressions (35), (37) and (38), which are obtained by the solution of formula (8) in boundary and initial conditions (33)-(34).

For a computation of the natural resources of unconfined ground water, one may use formulas (23), (33) and (38), in order to do this one must know the character of the fluctuation of the ground water level in a point of the recharge area of the water horizon.

It is advisable to approximate the natural boundary conditions, given as empiric functions, with Chebyshev polynominals. 